\title{
Ability of a confocal scanning laser ophthalmoscope (TopSS) to detect early glaucomatous visual field defect
}

\author{
Byoung-Sun Ahn, Changwon Kee
}

\begin{abstract}
Aims-To evaluate the ability of the confocal scanning laser ophthalmoscope (TopSS) to detect early glaucomatous visual field defect using our unique discriminant criteria.

Methods-The optic discs of 110 eyes of normal Korean subjects were examined and normal values for each variable were obtained according to the size of the optic disc. The five most sensitive optic disc variables for discriminating glaucoma were then applied to one eye of 80 Korean subjects with primary open angle glaucoma or normal tension glaucoma. Only eyes with an optic disc size of $2.0-3.0 \mathrm{~mm}^{2}$ and a contour tilt of less than $3^{\circ}$ were included. These variables were used to develop unique discriminant criteria for detecting early glaucomatous visual field defect and their sensitivity and specificity were calculated in three groups of patients with visual field loss.

Results-The five most sensitive variables were half the depth area, cup/disc (C/D) ratio, total area of the neuroretinal rim (NRR), volume above, and localised thinning of the NRR. The following criteria were used to diagnose glaucoma: (1) total area of the NRR decreased and one of the other four variables abnormal, and (2) total area of the NRR normal, localised thinning of the NRR, and one of the other three variables abnormal. The sensitivity of these criteria was $89.7 \%$ in patients with a mildly impaired visual field and $100 \%$ in those with a moderately or severely impaired visual field; the specificity was $89.1 \%$.

Conclusions-The discriminant criteria used had high sensitivity and specificity in the diagnosis of glaucoma and the TopSS can be useful in the early detection of changes in the glaucomatous optic disc. (Br F Ophthalmol 2000;84:852-855)
\end{abstract}

Ophthalmology, Samsung Medical Center, School of

Medicine,

Sungkyunkwan

University, 50

Ilwon-dong,

Kangnam-ku, Seoul

135-710, Korea

B-S Ahn

C Kee

Correspondence to: Dr C Kee

cwkee@smc.samsung.co.kr

Accepted 25 April 2000 laser ophthalmoscopy is the most advanced method in current use and provides objective, reproducible, quantitative, and three dimen- sional analysis of the optic disc. ${ }^{4-6}$ However, its usefulness is limited because there is considerable overlap between normal and glaucomatous eyes and by the fact that some optic disc variables are influenced by race $^{78}$ and by the size of the disc. ${ }^{3-11}$ Consequently, it is used mainly to monitor the progression of glaucoma rather than in its diagnosis. ${ }^{12-14}$ To overcome these limitations, race specific normal values of optic disc variables according to disc size are essential.

In this study we have obtained the normal values of optic disc variables of Koreans according to the disc size and evaluated the TopSS, a confocal scanning laser ophthalmoscope, in detecting early glaucomatous defects using our unique discriminant criteria.

Subjects and methods

One hundred and ten normal Korean adults visiting Samsung Health Promotion Center for routine physical examination and 80 patients diagnosed with primary open angle glaucoma or normal tension glaucoma from the Glaucoma Clinic in the Samsung Medical Center were enrolled in the study. Only one eye of each subject was studied.

Normal subjects were selected for the study providing they had no abnormal ocular findings on slit lamp examination; intraocular pressure less than $21 \mathrm{~mm} \mathrm{Hg}$ by non-contact air puff tonometer (Topcon CT-50); and normal visual field by Goldmann perimeter. Patients with primary open angle glaucoma met the following criteria: open and normal angle structure by gonioscopic examination; intraocular pressure more than $21 \mathrm{~mm} \mathrm{Hg}$ by Goldmann applanation tonometry before treatment; and glaucomatous visual field defect on the Humphrey field analyser (C30-2) in more than two consecutive tests. Patients with normal tension glaucoma had the same entry criteria as those with primary open angle glaucoma except that the intraocular pressure was below $21 \mathrm{~mm} \mathrm{Hg}$ by Goldmann applanation tonometry. Patients with glaucoma were excluded if they had maculopathy, vascular disorder, non-glaucomatous optic neuropathy, or media opacity to minimise the aberrant effect on the visual field.

The glaucoma patients were divided into three groups according to the mean deviation in global indices of the Humphrey visual field: (1) mildly impaired group with mean deviation of $<6 \mathrm{~dB}$; moderately impaired group with mean deviation of $6-12 \mathrm{~dB}$; and severely impaired group with mean deviation of 
Table 1 Demographic data

\begin{tabular}{lllll}
\hline & & \multicolumn{2}{l}{ Glaucomatous eyes } \\
\cline { 3 - 5 } & Normal eyes & Group I & Group II & Group III \\
\hline No & 110 & 29 & 19 & 32 \\
Mean (SD) age (years) & $52.6(5.8)$ & $51.2(7.9)$ & $54.9(9.1)$ & $48.5(9.8)$ \\
Sex (M:F) & $59: 51$ & $16: 13$ & $8: 11$ & $15: 17$ \\
\hline
\end{tabular}

Group I = mild visual field loss (mean deviation better than $-6 \mathrm{~dB}$ ); Group II = moderate visual field loss (mean deviation -6 to $-12 \mathrm{~dB}$ ); Group III = severe visual field loss (mean deviation worse than $-12 \mathrm{~dB})$.

Table 2 Normal values of eight optic disc variables according to optic disc size

\begin{tabular}{|c|c|c|}
\hline \multirow[b]{2}{*}{ Variables } & \multicolumn{2}{|c|}{ Normal values } \\
\hline & Group I & Group II \\
\hline Cup shape (lower limit) & -0.090 & -0.108 \\
\hline Cup area $\left(\mathrm{mm}^{2}\right)$ (upper limit) & 1.405 & 1.619 \\
\hline Half depth area $\left(\mathrm{mm}^{2}\right)$ (upper limit) & 0.649 & 0.779 \\
\hline $\mathrm{C} / \mathrm{D}$ ratio (upper limit) & 0.554 & 0.569 \\
\hline Total area of NRR $\left(\mathrm{mm}^{2}\right)$ (lower limit) & 1.000 & 1.113 \\
\hline Sector 6 & 0.114 & 0.130 \\
\hline Sector 12 & 0.112 & 0.130 \\
\hline Volume above $\left(\mathrm{mm}^{3}\right)$ (lower limit) & 0.124 & 0.157 \\
\hline Volume below $\left(\mathrm{mm}^{3}\right)$ (lower limit) & -0.488 & -0.572 \\
\hline Half depth volume $\left(\mathrm{mm}^{3}\right)$ (lower limit) & -0.136 & -0.140 \\
\hline
\end{tabular}

Group I $=2.0 \mathrm{~mm}^{2} \leqslant$ optic disc size $<2.5 \mathrm{~mm}^{2}$; Group II $=2.5 \mathrm{~mm}^{2}<$ optic disc size $\leqslant 3.0 \mathrm{~mm}^{2}$; $\mathrm{C} / \mathrm{D}$ ratio $=$ cup to disc ratio; $\mathrm{NRR}=$ neuroretinal rim.

$>12 \mathrm{~dB} .{ }^{15}$ All groups were age matched with normal subjects.

The optic disc variables were obtained by confocal scanning laser ophthalmoscopy using the TopSS ophthalmoscope (Laser Diagnostic Technologies Inc, San Diego, CA, USA) by one examiner. The TopSS automatically provided the following 14 optic disc variables ${ }^{11}$ : contour variation, mean contour depth, cup shape, cup area, half depth area, cup/disc (C/D) ratio, area of neuroretinal rim (NRR), volume above, volume below, half depth volume, average depth, maximum depth, average slope, and maximum slope. The NRR area, one of the 14 variables, was divided into 12 sectors and the value of each sector was also measured. Baseline images were made using the average of three consecutive images. Only eyes with an optic disc in the size range $2.0-3.0 \mathrm{~mm}^{2}$ were included. Eyes with an optic disc contour tilt of more than $3^{\circ}$ were excluded to avoid inclusion of eyes with high myopia. The cup margin was

Table 3 Normal values of six optic disc variables not correlated with optic disc size

\begin{tabular}{ll}
\hline Variables & Normal values \\
\hline Contour variation (lower limit) & 0.253 \\
Mean contour depth (mm) (lower limit) & -0.410 \\
Average depth (mm) (lower limit) & -0.377 \\
Maximum depth (mm) (lower limit) & -0.967 \\
Average slope $\left(^{\circ}\right.$ (upper limit) & 39.10 \\
Maximum slope $\left(^{\circ}\right)$ (upper limit) & 85.95 \\
\hline
\end{tabular}

Table 4 Abnormal ratio of optic disc variables according to glaucomatous field defect

\begin{tabular}{llll}
\hline \multirow{2}{*}{ Variables } & \multicolumn{2}{l}{ Abnormal ratio, no (\%) } \\
\cline { 2 - 4 } & Group I & Group II & Group III \\
\hline Half depth area & $19 / 29(65.5)$ & $14 / 19(73.7)$ & $26 / 32(81.25)$ \\
C/D ratio & $23 / 29(79.3)$ & $19 / 19(100)$ & $32 / 32(100)$ \\
Total area of NRR & $22 / 29(75.9)$ & $19 / 19(100)$ & $32 / 32(100)$ \\
Volume above & $15 / 29(51.7)$ & $16 / 19(84.2)$ & $23 / 32(71.9)$ \\
Localised thinning of NRR & $19 / 29(65.5)$ & $16 / 19(84.2)$ & $31 / 32(96.9)$ \\
\hline
\end{tabular}

Group I = mild visual field loss (mean deviation better than $-6 \mathrm{~dB}$ ); Group II = moderate visual field loss (mean deviation -6 to $-12 \mathrm{~dB}$ ); Group III = severe visual field loss (mean deviation worse than $-12 \mathrm{~dB}) ; \mathrm{C} / \mathrm{D}$ ratio $=$ cup to disc ratio; $\mathrm{NRR}=$ neuroretinal rim. defined at $100 \mu \mathrm{m}$ below the retinal reference plane. Scan angle was set at $10^{\circ}$ and scan depth and scan offset were adjusted at $3 \mathrm{~mm}$ and 3, respectively. The optic disc margin was defined by changing the shape of ellipse and fitting the best ellipse around the scleral ring.

Normal values of optic disc variables were obtained from normal subjects according to the disc size. The measurements between the 5 th and 95 th percentiles were regarded as normal. The abnormal ratio of each variable in each of the three glaucoma groups was obtained and five highly sensitive diagnostic variables, with abnormal rates of more than $50 \%$, were determined. These diagnostic variables were: half depth area (area of all points half way between the cup margin and the bottom); C/D ratio calculated by dividing the cup area by the total optic disc area; total area of NRR (difference between total disc area and cup area); volume above (the volume of all tissues within the NRR area); and localised thinning of the NRR (decreased NRR area at 6 or 12 o'clock location). We then established the discriminant criteria using these five variables and analysed the sensitivity and specificity of these discriminant criteria according to the severity of the visual field defect in the patients with glaucoma.

Statistical analysis on difference in age and sex between the normal subjects and glaucoma patients was performed using the Student's $t$ test.

\section{Results}

There was no significant difference in age and sex between the normal subjects and patients with glaucoma $(p>0.1)$. Of the 80 eyes in the patients with glaucoma, 29 were mildly impaired, 19 were moderately impaired, and 32 were severely impaired (Table 1).

The 110 eyes of the normal subjects were divided into two groups according to the optic disc size; 53 had a disc size of $2.0-2.5 \mathrm{~mm}^{2}$ and the remaining 57 eyes had a disc size larger than $2.5-3.0 \mathrm{~mm}^{2}$. The normal values influenced by the optic disc size are listed in Table 2 and those not influenced by the optic disc size are shown in Table 3.

Applying the normal values of these variables to the eyes of the patients with glaucoma, variables such as half depth area, C/D ratio, total area of NRR, volume above, and localised thinning of NRR - which were all influenced by the size of the optic disc-had an abnormal ratio in more than $50 \%$ (Table 4 ). The abnormal ratio had a tendency to increase as the severity of the visual field defect increased. Using these variables, the following discriminant criteria for detecting glaucoma were made: (1) decreased total area of NRR and one of the other four variables abnormal, and (2) normal total area of NRR, localised thinning of NRR, and one of the other three variables abnormal.

The sensitivity of these diagnostic criteria was $89.7 \%$ in patients with a mildly impaired visual field and $100 \%$ in those in whom the visual field was moderately or severely impaired. The specificity was $89.1 \%$. 


\section{Discussion}

Evaluation of the optic disc is more important than the visual field test in the early detection of glaucoma because it has been reported that changes in the optic disc precede the visual field defect. ${ }^{16-19}$ However, the optic disc has features which vary widely between normal and glaucomatous eyes, hence subtle changes in the optic disc in patients with glaucoma are not easily detected, even by an expert. ${ }^{20}$ Comparison of serial stereophotography of the optic disc is objective and has been recommended as a convenient method for the early diagnosis of glaucoma. ${ }^{17} 2122$ However, a more accurate, quantitative, and three dimensional measurement is needed. It has been reported that defining the disc area is more reproducible than defining the cup area. ${ }^{23}$ This suggests that an automated optic disc analysis in which only the disc area is defined by the examiner and the other variables are calculated by computer software is more objective and valuable. The confocal scanning laser ophthalmoscope, which can analyse minute structural changes, fulfils these requirements and is superior to any other tool. It is useful in detecting the progression of glaucoma. ${ }^{12-14}$ To be useful in the diagnosis of glaucoma it is necessary to estimate the normal values and to determine which optic disc variables are more sensitive to glaucomatous change.

Many studies have attempted to find the most powerful variable of early changes in the glaucomatous optic disc, ${ }^{24-26}$ but they have not taken into account factors influencing the optic disc variables. The size of the optic disc is one of these factors. The larger the optic disc, the larger the cup, the neural rim, and the optic nerve fibre count. ${ }^{3-11}$ Ethnicity is another factor in optic disc analysis. ${ }^{78} \mathrm{~A}$ study with the Heidelberg retina tomograph by Iester et al recently reported that a discriminant analysis formula composed of three variables had a sensitivity of $78.7 \%$ and a specificity of $89.7 \%$ in eyes with an optic disc size in the range $2.0-3.0 \mathrm{~mm}^{2} .{ }^{27}$ The three variables used were cup shape measure, rim volume, and height variation contour which they had found to be useful in the detection of early glaucomatous change in their previous study. ${ }^{28}$ However, height variation contour may be highly variable according to the optic disc border determined by the examiner, and it has been reported that the cup shape measure is not a good variable to indicate change in the glaucomatous optic disc. $^{29} 30$

We have therefore used other discriminant variables such as the $\mathrm{C} / \mathrm{D}$ ratio, volume above, half depth area, total area of NRR, and localised thinning of NRR, which had good sensitivity in discriminating changes in the optic disc of patients with glaucoma. The abnormal C/D ratio and total area of NRR was large even in patients with a mildly impaired visual field and increased as the visual field defect progressed, indicating that they are good indicators of change in the glaucomatous optic disc. Interestingly, the abnormal ratio of volume above was larger in those with a moderately impaired visual field than in those in whom the visual field was severely impaired. We postulate that the volume of NRR decreases as the glaucoma progresses. However, when the glaucoma is so severe that the nerve fibre layer on the retina has thinned, this causes the retinal reference plane to set deeper and thereby the volume above may appear inadvertently to increase.

Localised thinning of the NRR is also a sensitive marker of glaucomatous change since the glaucomatous cupping usually begins at the superior or inferior pole of the disc. ${ }^{31}$ In this study we divided the area of the NRR into 12 sectors and calculated the abnormal ratio in each sector. The abnormal ratio was large and proportional to the severity of the visual field defect at the 6 o'clock and 12 o'clock locations of the NRR. Diffuse cup enlargement and focal changes of the NRR rim area in the superior and inferior poles, which were described in this study as the decreased total area of the NRR and localised thinning of the NRR, respectively, are the most common early changes found in patients with glaucoma. ${ }^{182232}$ Our discriminant criteria fulfilled the above findings and took into consideration race specificity and optic disc size.

Our discriminant criteria were selected on the assumption that a combination of several sensitive variables would achieve better results than a single variable in the early detection of changes in the optic disc in patients with glaucoma. They are simple, but can be clinically useful in the diagnosis of early glaucomatous change. Furthermore, since the volume and area of the NRR are not significantly different between ethnic groups, ${ }^{8}$ these criteria may be of use as a reference in other ethnic groups. The sensitivity and specificity of these criteria were $89.7 \%$ and $89.1 \%$, respectively. This means that about $10 \%$ of subjects could be misdiagnosed as having or not having glaucoma. We included only those with optic discs tilted less than $3^{\circ}$ and of size range 2.0$3.0 \mathrm{~mm}^{2}$. Since it is still not easy to evaluate tilted discs, and it takes time to include enough smaller or larger discs to establish the normal range in these disc sizes, analysis of the tilted disc and variables in various disc sizes still remains a challenge.

In conclusion, the discriminant criteria used in this study had a high sensitivity and specificity in the diagnosis of glaucoma and the confocal scanning laser ophthalmoscope, the TopSS, can be useful in the early diagnosis of changes in the optic disc of patients with glaucoma.

Funding: This study was supported by grant SMC98 from the Samsung Medical Center, Seoul, Korea.

1 Spencer AF, Vernon SA. Optic disc measurement: a comparison of indirect ophthalmoscopic methods. $\mathrm{Br} f$ Ophthalmol 1995;79:910-5.

2 Pashby RC, MacDonald RK. Photographic assessment of the optic disc. Can f Ophthalmol 1975;10:286-9.

3 Britton RJ, Drance SM, Schulzer M, et al. The area of the neuroretinal rim of the optic nerve in normal eyes. Am $\mathcal{F}$ Ophthalmol 1987;103:497-504.

4 Chihara E, Takahashi F, Chirara K. Assessment of optic disc topography with scanning laser ophthalmoscope. Graefes
Arch Clin Exp Ophthalmol 1993;231:1-6.

5 Woon WH, Fitzke FW, Bird AC, et al. Confocal imaging of the fundus using a scanning laser ophthalmoscope. $B r f$ Ophthalmol 1992;76:470-4. 
6 Dreher AW, Tso PC, Weinreb RN. Reproducibility of topographic measurements of the normal and glaucomatous optic nerve head with laser

7 Chi T, Ritch R, Stickler D, et al. Racial differences in optic nerve head parameters. Arch Ophthalmol 1989;107:836-9.

8 Tsai CS, Zangwill L, Gonzalez C, et al. Ethnic differences in optic nerve head topography. F Glaucoma 1995;4:248-57.

9 Jonas JB, Schmidt AM, Müller-Bergh JA, et al. Human optic nerve fiber count and optic disc size. Invest Ophthalmol Vis Sci 1992;33:2012-8.

10 Quigley HA, Coleman AL, Dorman-Pease ME. Large optic nerve heads have more nerve fibers in normal monkey eyes. Arch Ophthalmol 1991;109:1441-3.

11 Kee C, Koo H, Ji Y. Effect of optic disc size or age on evaluation of optic disc variables. Br f Ophthalmol 1997;81: 1046-9.

12 Kruse FE, Burk ROW, Völcker HE, et al. Reproducibility of topographic measurements of the optic nerve head with
laser tomographic scanning. Ophthalmology 1989;96:13204 .

13 Rohrschneider K, Burk ROW, Kruse FE, et al. Reproducibility of the optic nerve head topography with a new laser tomographic scanning device. Ophthalmology 1994;101: 1044-9.

14 Weinreb RN. Diagnosing and monitoring glaucoma with confocal scanning laser ophthalmoscopy. F Glaucoma 1995 4:225-7.

15 Hodapp E, Parrish RK II, Anderson DR. Clinical decisions in glaucoma. 1st ed. St Louis: Mosby-Year Book, 1993: 53-61.

16 Pederson JE, Anderson DR. The mode of progressive disc cupping in ocular hypertension and glaucoma. Arch Ophthalmol 1980;98:490-5.

17 Odberg T, Riise D. Early diagnosis of glaucoma. The value of successive stereophotography of the optic disc. Acta Ophthalmol 1985;63:257-63.

18 Quigley HA, Addicks EM, Green WR. Optic nerve damage in human glaucoma. Arch Ophthalmol 1982;100:35-46.

19 Zeyen TG, Caprioli J. Progression of disc and field damage in early glaucoma. Arch Ophthalmol 1993;111:62-5.
20 Lichter PR. Variability of expert observers in evaluating the optic disc. Trans Am Ophthalmol Soc 1976;74:532-72.

21 Sommer A, Pollack I, Maumenee AE. Optic disc parameters and onset of glaucomatous field loss. Arch Ophthalmol 1979;97:1444-8.

22 Kirsch RE, Anderson DR. Clinical recognition of glaucomatous cupping. Am f Ophthalmol 1973;75:442-54.

23 Bengtsson B. The variation and covariation of cup and disc diameters. Acta Ophthalmol 1976;54:804-18.

24 Uchida H, Brigatti L, Caprioli J. Detection of structural damage from glaucoma with confocal laser image analysis. Invest Ophthalmol Vis Sci 1996;37:2393-401.

25 Brigatti L, Caprioli J. Correlation of visual field with scanning confocal laser optic disc measurements in glaucoma. Arch Ophthalmol 1995;113:1191-4.

26 Geyer O, Michaeli-Cohen A, Silver DM, et al. Reproducbility of topographic measures of the glaucomatous opti nerve head. Br f Ophthalmol 1998;82:14-7.

27 Iester M, Mikelberg FS, Drance SM. The effect of optic disc size on diagnostic precision with the Heidelberg Retina Tomograph. Ophthalmology 1997;104:545-8.

28 Mikelberg FS, Parfitt CM, Swindale NV, et al. Ability of the Heidelberg Retina Tomograph to detect early glaucomatous visual field loss. F Glaucoma 1995;4:242-7.

29 Wollstein G, Garway-Heath DF, Hitchings RA. Identification of early glaucoma cases with the scanning laser ophthalmoscope. Ophthalmology 1998;105:1557-63.

$30 \mathrm{Kamel}$ DS, Viswanathan AC, Garway-Heath DF, et al. Detection of optic disc change with the Heidelberg retina tomograph before confirmed visual field change in ocular hypertensives converting to early glaucoma. $\mathrm{Br} \mathcal{F}$ Ophthalmol 1999;83:290-4

31 Airaksinen PJ, Tuulonen A, Werner EB. Clinical evaluation of the optic disc and retinal nerve fiber layer. In: Ritch R, Shields MB, Krupin T, eds. The glaucomas. 2nd ed. St Louis: Mosby, 1996: 631-3.

32 Tuulonen A, Airaksinen PJ. Initial glaucomatous optic disk and retinal nerve fiber layer abnormalities and their progression. Am f Ophthalmol 1991;111:485-91. 